\title{
INVARIANCE OF FLUID LIMITS FOR THE SHORTEST REMAINING PROCESSING TIME AND SHORTEST JOB FIRST POLICIES
}

\author{
H. Christian Gromoll*and Martin Keutel \\ University of Virginia
}

November 21, 2018

\begin{abstract}
We consider a single-server queue with renewal arrivals and i.i.d. service times, in which the server employs either the preemptive Shortest Remaining Processing Time (SRPT) policy, or its non-preemptive variant, Shortest Job First (SJF). We show that for given stochastic primitives (initial condition, arrival and service processes), the model has the same fluid limit under either policy. In particular, we conclude that the well-known queue length optimality of preemptive SRPT is also achieved, asymptotically on fluid scale, by the simpler-toimplement SJF policy. We also conclude that on fluid scale, SJF and SRPT achieve the same performance with respect to response times of the longest-waiting jobs in the system.
\end{abstract}

AMS 2010 subject classifications. Primary 60K25, 60F17; secondary 60G57, 68M20, 90B22.

Key words. Queueing, queue length, shortest remaining processing time, shortest job first, shortest job next, fluid limit.

\section{Introduction}

Schrage's [10] classic result asserts that for given stochastic primitives (initial condition, arrival and service processes), the preemptive Shortest Remaining Processing Time (SRPT) policy minimizes queue length at all times, over all (work-conserving) policies. Recently, Gromoll, Kruk, and Puha [6] showed that, in the heavy-traffic regime, the diffusion scaled queue length process under SRPT also achieves the optimal lower bound $W(t) / x^{*}$ for all $t \geq 0$,

${ }^{*}$ Research supported in part by NSF grant DMS 0707111 
where $W(\cdot)$ is the diffusion limit of the workload process and $x^{*}$ is the supremum of the support of the service time distribution.

Under SRPT, preemptive priority is given to the job that can be completed first. More precisely, consider a single server queue with renewal arrivals and i.i.d. service times, and let $\mathcal{I}(t)$ index, in arrival order, those jobs that are in the queue at time $t$. For a job $i \in \mathcal{I}(t)$, let $v_{i}(t)$ denote its residual service time at time $t$, or the remaining amount of processing time required to complete this job. If $j \in \mathcal{I}(t)$ is the smallest index such that $v_{j}(t) \leq v_{i}(t)$ for all $i \in \mathcal{I}(t)$, then under SRPT, $\frac{d}{d t} v_{j}(t+)=-1$ and $\frac{d}{d t} v_{i}(t+)=0$ for all $i \in \mathcal{I}(t) \backslash j$.

Due to its simple description and attractive optimality properties (see also Smith [12]), the SRPT policy and its relatives have received much attention over the past several decades; see Schreiber [11] for a survey, as well as [2, 4] and references therein for more recent work.

Despite its theoretical advantages, SRPT may not always be the best choice in practice. Practical disadvantages include inexact job size information, the need to continuously monitor the state of the queue and keep this state as a continuum of priority classes, inefficiencies associated with frequent switchovers, and long response times for large jobs. See for example [1, 13.

These disadvantages have led researchers to consider a number of variants of SRPT, all of which retain the essential feature of prioritizing smaller jobs in some way. Care is needed when reviewing the literature, as differing terminology is in use. Usually, "SRPT" refers to the preemptive policy defined above. This policy may also be referred to as Shortest Time to Completion First, or STCF.

Shortest Job First (SJF) is the non-preemptive variant of SRPT. An SJF server still selects the smallest job in the queue. But once begun, this job is served to completion before the server makes another selection. This policy may also be referred to as Shortest Job Next (SJN). There are also preemptive versions of SJF and SJN, which differ from SRPT because they prioritize based on initial rather than remaining job size. Nuyens and Wierman [13] have introduced the class of $\epsilon$-SMART policies, which include the preemptive policies mentioned above as well as policies that allow for inexact job size information and finite sets of priority classes based on job size.

In this paper, we compare the preemptive SRPT policy to its nonpreemptive SJF variant. Our analysis implies that, in many respects, these two policies achieve the same performance (although not in all respects; see 
below). Although SJF does not resolve all of the criticisms that have been raised for SRPT, it does have important advantages from an implementation stand point. In particular, there is no need to continuously monitor the state of the queue and it avoids any penalties for interrupting service. Consequently, practitioners interested in performance measures for which there is no essential difference between the two policies may opt for the simpler SJF policy, and this paper provides the theoretical justification for such a choice.

\subsection{Main result}

Consider an $r$-indexed sequence of single server queues, each with renewal arrivals, generally distributed i.i.d. service times, and random initial state. In the $r$ th model, let $\mathcal{Z}_{p}^{r}(\cdot)$ denote the measure-valued state descriptor when the server employs preemptive SRPT, and let $\mathcal{Z}^{r}(\cdot)$ denote the state descriptor under SJF. These processes track the residual service times of all jobs, as they evolve according to each respective policy; see Section 2 for details. Let $\overline{\mathcal{Z}}_{p}^{r}(\cdot)$ and $\overline{\mathcal{Z}}^{r}(\cdot)$ denote these processes under fluid scaling. We will impose the standard asymptotic assumptions (28)-(32) of Section 4. In particular, there is a limiting random initial measure $\mathcal{Z}_{0}$, a limiting arrival rate $\alpha$, and a limiting service time distribution $\nu$, such that the limiting traffic intensity is at most one.

In this setting, Down, Gromoll, and Puha [4] showed that as $r \rightarrow \infty$, the sequence $\left\{\overline{\mathcal{Z}}_{p}^{r}(\cdot)\right\}$ converges in distribution to a process $\mathcal{Z}^{*}(\cdot)$ that is almost surely an SRPT fluid model solution for the data $(\alpha, \nu)$ and initial condition $\mathcal{Z}^{*}(0)$, where $\mathcal{Z}^{*}(0)=\mathcal{Z}_{0}$ in distribution; see Theorem 3.9 in [4].

An SRPT fluid model solution for $(\alpha, \nu)$ with initial condition $\xi$ is a deterministic measure-valued function $\zeta(\cdot)$ satisfying $\zeta(0)=\xi$ and a certain family of dynamic inequalities; see Section 2.2, Definition 2.2 in [4]. Properties of SRPT fluid model solutions were extensively studied in [4].

The main result of this paper is that the sequence of state descriptors under the SJF policy has the same fluid limit:

Theorem 1. Under the asymptotic assumptions (28)-(32), the sequence $\left\{\overline{\mathcal{Z}}^{r}(\cdot)\right\}$ converges in distribution to the measure valued process $\mathcal{Z}^{*}(\cdot)$, where $\mathcal{Z}^{*}(\cdot)$ is almost surely an SRPT fluid model solution for the data $(\alpha, \nu)$ and initial condition $\mathcal{Z}^{*}(0)$, and $\mathcal{Z}^{*}(0)$ is equal in distribution to $\mathcal{Z}_{0}$. 


\subsection{Implications for comparing performance}

Since the queue length processes under SJF and SRPT are given by the total mass of the state descriptors $\mathcal{Z}^{r}(\cdot)$ and $\mathcal{Z}_{p}^{r}(\cdot)$ respectively, an immediate consequence of the above theorem is that, asymptotically on fluid scale, SJF enjoys the same queue length optimality as SRPT.

A second consequence relates to state-dependent response times. A job's state-dependent response time is the time until it exits the system, conditional on the state of the system (the configuration of all current residual service times) when it arrives. The fluid limit $\mathcal{Z}^{*}(\cdot)$ can be used to calculate a fluid approximation $s(x)$ to the state-dependent response time of a job of size $x$, as a function of an initial state $\xi$; see [5, 4. Thus Theorem 1 implies that, asymptotically on fluid scale, a job of size $x$ arriving to a queue in state $\xi$ will have the same state-dependent response time under SJF or SRPT. Moreover, the system will be in the same state $\xi$ under either SJF or SRPT.

Note that SJF and SRPT exhibit differing performance for the mean response time of a job of size $x$ (that is, averaged over all possible states encountered on arrival when the system is in steady state). Bansal and Harchol-Balter [2] show for heavy-tailed service time distributions, that the mean response time under SRPT is orders of magnitude lower than under SJF, while the very largest jobs have smaller mean response times under SJF. See also Harchol-Balter, Sigman, and Wierman [7] for explicit formulae for mean response times under SRPT, SJF, and several other protocols.

The reason SJF and SRPT have the same fluid approximation for statedependent response times, is that fluid scaling compresses time such that individual jobs - even large ones - exit almost instantaneously once they begin service. In fact, any arriving job with service time smaller than the largest job to begin service during the current busy period, or frontier, will have negligible response time on fluid scale. Jobs with service times larger than the frontier wait a positive time on fluid scale to begin service for the first time.

Thus, for either policy, the fluid limit shows that response times for jobs larger than the frontier are orders of magnitude longer than for those below it. In particular, the fluid limit captures the profile of reponse times only for those jobs that experience the very longest response times in the system, namely, those that are bigger than the frontier when they arrive. And given the initial state, these response times are the same under SJF or SRPT. This conclusion is consistent with Nuyens and Zwart [8], where it is shown that the steady-state response time has the same asymptotic decay rate under 
SJF and SRPT.

\subsection{Notation}

For real numbers $a$ and $b$, let $a \wedge b=\min \{a, b\}$ and $a \vee b=\max \{a, b\}$. Denote the natural numbers $1,2, \ldots$ by $\mathbb{N}$ and the non-negative real numbers $[0, \infty)$ by $\mathbb{R}_{+}$. The measure that puts one unit of mass at $x \in \mathbb{R}_{+}$is written $\delta_{x}$, and the meaure $\delta_{x}^{+}$is $\delta_{x}$ if $x>0$ and the zero measure (denoted $\mathbf{0}$ ) if $x=0$.

Let $\mathbf{M}$ denote the Polish space of finite, nonnegative Borel measures on $\mathbb{R}_{+}$, endowed with the weak topology [9]. A metric inducing this topology is the following Prohorov metric on M. For $A \subset \mathbb{R}_{+}$, let $A^{\epsilon}=\left\{y \in \mathbb{R}_{+}\right.$: $\left.\inf _{x \in A}|x-y|<\epsilon\right\}$. Then for $\xi, \zeta \in \mathbf{M}$, define

$$
\begin{aligned}
\mathbf{d}[\xi, \zeta]=\inf \left\{\epsilon>0: \xi(B) \leq \zeta\left(B^{\epsilon}\right)+\epsilon \text { and } \zeta(B)\right. & \leq \xi\left(B^{\epsilon}\right)+\epsilon \\
& \text { for all closed } \left.B \subset \mathbb{R}_{+}\right\} .
\end{aligned}
$$

For $\zeta \in \mathbf{M}$ and a $\zeta$-integrable function $g: \mathbb{R}_{+} \rightarrow \mathbb{R}$, define $\langle g, \zeta\rangle=$ $\int_{\mathbb{R}_{+}} g(x) \zeta(d x)$. Let $\chi(x)=x$ be the identity function, and for any set $A$, let $1_{A}$ denote its indicator function. For a real-valued function $f$, write $f^{+}$ and $f^{-}$for its positive and negative parts respectively. Unless otherwise specified, all processes are assumed to be right continuous with finite left limits (rcll). In particular, all measure-valued processes in this paper take values in the Skorohod space $\mathbf{D}([0, \infty), \mathbf{M})$ endowed with the $J_{1}$-topology.

Convergence in probability is denoted $\stackrel{\mathbf{P}}{\longrightarrow}$, weak convergence of elements of $\mathbf{M}$ is denoted $\stackrel{w}{\longrightarrow}$, and convergence in distribution of random objects is denoted $\Rightarrow$. We adopt the convention that a sum of the form $\sum_{i=n}^{m}$ with $n>m$, or a sum over an empty set of indices equals zero. Finally, we also define $\min \varnothing=\inf \varnothing=\infty$.

\section{The model: SJF and SRPT}

This section lays out the precise definitions of our model, including the two policies under consideration. We will compare the SJF and SRPT policies pathwise, using the same sample space $(\Omega, \mathcal{F}, \mathbf{P})$ and same stochastic primitives. The stochastic primitives consist of an exogenous arrival process, a sequence of service times, and an initial condition.

Stochastic primitives. The arrival process $E(\cdot)$ is a rate $\alpha \in(0, \infty)$ (possibly delayed) renewal process. For $t \geq 0, E(t)$ is the number of jobs that have arrived to the queue during $(0, t]$. The initial service times of these 
jobs are taken from an i.i.d. sequence $\left\{w_{j}: j=1,2, \ldots\right\}$ of strictly positive random variables, with distribution $\nu$ having finite mean. Define the traffic intensity $\rho=\alpha\langle\chi, \nu\rangle$.

The initial condition is given by a non-negative integer-valued random variable $Z_{0}$ with finite mean, and a sequence of strictly positive random variables $\left\{w_{j}: j=\ldots,-2,-1\right\}$. The random variable $Z_{0}$ represents the number of jobs in the queue at time 0 , and their initial service times are $\left\{w_{-Z_{0}}, \ldots, w_{-1}\right\}$.

Define the random index set $J=\left\{j \in \mathbb{Z}: j \geq-Z_{0}\right\} \backslash\{0\}$ and, for each $t \geq 0$, define the random index set $J(t)=J \cap\left\{-Z_{0}, \ldots, E(t)\right\}$. Then $J$ indexes all jobs that will ever be in the system, and $J(t)$ indexes all jobs that have been in the system by time $t$. Note that neither set ever contains the index 0 , and that $J(t)=\varnothing$ if $Z_{0}=E(t)=0$. Note that the present indexing differs slightly from that used in [4], but this does not affect our analysis.

Workload. Define a measure-valued load process $\mathcal{V}(\cdot)$ and a load process $V(\cdot)$ by

$$
\begin{aligned}
\mathcal{V}(t) & =\sum_{j=1}^{E(t)} \delta_{w_{j}}, \\
V(t) & =\langle\chi, \mathcal{V}(t)\rangle,
\end{aligned}
$$

for all $t \geq 0$. Define the workload process $W(\cdot)$ and cumulative idle time process $I(\cdot)$ by

$$
\begin{aligned}
W(0) & =\sum_{j=-Z_{0}}^{-1} w_{j}, \\
I(t) & =\sup _{s \leq t}[W(0)+V(s)-s]^{-}, \\
W(t) & =W(0)+V(t)-t+I(t) .
\end{aligned}
$$

Next, we define the SJF and SRPT policies, as well as the corresponding state descriptors that describe the evolution of the system under each policy.

Shortest Job First. In order to define the residual service times under SJF, we first define the succesive times $\left\{\gamma_{n}\right\}$ at which the server begins processing a new job. Let $\gamma_{0}=y_{0}=j_{0}=0$ and define the set $J_{0}=\varnothing$. Now 
define inductively, for $n=1,2, \ldots$,

$$
\begin{aligned}
\gamma_{n} & =\inf \left\{t \geq \gamma_{n-1}+y_{n-1}: Z_{0}+E(t) \geq n\right\}, \\
y_{n} & =\min \left\{w_{j}: j \in J\left(\gamma_{n}\right) \backslash J_{n-1}\right\}, \\
j_{n} & =\min \left\{j \in J\left(\gamma_{n}\right) \backslash J_{n-1}: w_{j}=y_{n}\right\}, \\
J_{n} & =J_{n-1} \cup\left\{j_{n}\right\} .
\end{aligned}
$$

For each $n=1,2, \ldots$, the time $\gamma_{n}$ is the $n$th time at which the server begins processing a new job, $y_{n}$ is the service requirement of this job, $j_{n}$ is its index, and $J_{n}$ is the set indexing all jobs that have begun service by time $\gamma_{n}$. For each $j \in J$, let

$$
n(j)=\min \left\{n \in \mathbb{N}: j \in J_{n}\right\} .
$$

Then $n(j)$ is the overall order in which job $j$ is served, and the start time of job $j$ may be defined as $\gamma_{n(j)}$ (with $\gamma_{\infty}$ interpreted as $\infty$, which may occur for example in a supercritical model in which job $j$ never begins service). In particular, note that $y_{n(j)}=w_{j}$ for all $j \in J$ such that $\gamma_{n(j)}<\infty$. At time $t \geq 0$, define the attained service of job $j \in J$ as

$$
s_{j}(t)=\left(t-\gamma_{n(j)}\right)^{+} \wedge w_{j},
$$

the cumulative service provided as

$$
S(t)=\sum_{j \in J(t)} s_{j}(t)
$$

and define the residual service time of job $j \in J$ as

$$
w_{j}(t)=w_{j}-s_{j}(t)
$$

We now define the state descriptor under SJF as the measure-valued process

$$
\mathcal{Z}(t)=\sum_{j \in J(t)} \delta_{w_{j}(t)}^{+}, \quad t \geq 0
$$

Finally, for each $t \geq 0$, let $F(t)$ denote the largest job started by the SJF server by time $t$. That is,

$$
F(t)=\max _{n \in \mathbb{N}}\left\{y_{n}: \gamma_{n} \leq t\right\} .
$$

Shortest Remaining Processing Time. For job $j \in J$, let $v_{j}(t)$ denote its residual service time at time $t$ when the server employs the SRPT 
policy; we refer the reader to [4] for a detailed definition. Then the measurevalued state descriptor under SRPT is given by

$$
\mathcal{Z}_{p}(t)=\sum_{j \in J(t)} \delta_{v_{j}(t)}^{+}, \quad t \geq 0
$$

Throughout the paper, the subscript $p$ for "preemptive" is used to distinguish performance processes under SRPT from their analogues under SJF. In [4], the left edge process of the state descriptor $\mathcal{Z}_{p}(\cdot)$ is defined as

$$
L_{p}(t)=\sup \left\{x \in \mathbb{R}_{+}:\left\langle 1_{[0, x)}, \mathcal{Z}_{p}(t)\right\rangle=0\right\}, \quad t \geq 0 .
$$

The current residual service time process is then defined, for all $t \geq 0$, by

$$
C_{p}(t)= \begin{cases}L_{p}(t), & \text { if } \mathcal{Z}_{p}(t) \neq \mathbf{0} \\ 0, & \text { otherwise },\end{cases}
$$

and the SRPT frontier process is defined by

$$
F_{p}(t)=\sup _{0 \leq s \leq t} C_{p}(s), \quad t \geq 0 .
$$

\section{Pathwise comparison}

In this section, we establish several results that relate SJF to SRPT on a pathwise basis. The main goal is to show that SJF and SRPT have the same frontier process almost surely. This will provide the key element in the proof of Theorem 1 in Section 4 ,

First, we show that the sum of the residual service times under SJF, as defined above, equals the workload defined in (11).

Lemma 1. Almost surely, for all $t \geq 0, S(t)=t-I(t)$, and $W(t)=$ $\sum_{j \in J(t)} w_{j}(t)$.

Proof. Clearly $S(t) \leq W(0)+V(t)$ for all $t \geq 0$. Recall that a regular point of a function is a point at which it is differentiable. We first show that, for all regular points $t$,

$$
\dot{S}(t)=0 \quad \text { if and only if } \quad S(t)=W(0)+V(t) .
$$

Note that almost surely for all $j \in J$, the derivative $\dot{s}_{j}(t) \in\{0,1\}$ for all regular points $t$ of $s_{j}(\cdot)$, and $\dot{s}_{j}(t)=1$ if and only if $t \in\left(\gamma_{n(j)}, \gamma_{n(j)}+y_{n(j)}\right)$. 
Further, the definitions above imply that the finite elements of $\{n(j): j \in J\}$ are distinct and the time intervals $\left\{\left(\gamma_{n}, \gamma_{n}+y_{n}\right): n \in \mathbb{N}\right\}$ are all disjoint. This implies that $\dot{S}(t) \in\{0,1\}$ for all regular points $t$. (Note that if $t$ is a regular point of $S(\cdot)$ but not of all $s_{j}(\cdot), j \in J(t)$, then we must have $\dot{S}(t)=1$ anyway). Moreover, $\dot{S}(t)=0$ if and only if $t<\gamma_{1}$ or $t \in\left(\gamma_{n}+y_{n}, \gamma_{n+1}\right)$ for some $n \in \mathbb{N}$. If $t<\gamma_{1}$, then $S(t)=0=W(0)+V(t)$, and the converse is also true. If $t \in\left(\gamma_{n}+y_{n}, \gamma_{n+1}\right)$ for some $n \in \mathbb{N}$, then (2) implies $Z_{0}+E(t)=n$, which implies that $s_{j}(t)=w_{j}$ for all $j \in J(t)$. Thus, $S(t)=W(0)+V(t)$, and the converse is also true. This proves (3).

Observe that almost surely, $S(\cdot)$ is continuous, starts at zero, has derivative in $\{0,1\}$ at regular points, is bounded above by $W(0)+V(\cdot)$, and satisfies (3) at all regular points $t$. Since $W(0)+V(\cdot)$ is right-continuous and piecewise constant, this uniquely determines $S(\cdot)$ almost surely.

As is well known, $\dot{I}(t) \in\{0,1\}$ at all regular points $t$, and $\dot{I}(t)=1$ if and only if $I(t)=(W(0)+V(t)-t)^{-}$and $I(t)>0$. So $\frac{d}{d t}(t-I(t))=0$ if and only if $t-I(t)=W(0)+V(t)$. Also, $t-I(t) \leq W(0)+V(t)$ for all $t \geq 0$. Since $\cdot-I(\cdot)$ is continuous and starts from zero, it is also uniquely determined by $W(0)+V(\cdot)$ almost surely. We conclude that $S(t)=t-I(t)$ for all $t \geq 0$ almost surely.

The second part follows directly from the first part and equation (1), since

$$
W(t)=W(0)+V(t)-t+I(t)=\sum_{j \in J(t)} w_{j}-S(t)=\sum_{j \in J(t)} w_{j}(t) .
$$

Note that by Lemma 1, for all $t \geq 0$,

$$
W(t)=\langle\chi, \mathcal{Z}(t)\rangle .
$$

Next, we prove a result describing the workload at or above the frontier under SJF, at certain random times. To that end, define the random set

$$
\mathcal{J}=\left\{j \in J: \gamma_{n(j)}<\infty \text { and } w_{j}=F\left(\gamma_{n(j)}\right)\right\} .
$$

These are frontier jobs, with service time equal to the frontier when they are started. The set of start times of frontier jobs is $\mathcal{T}=\left\{\gamma_{n(j)}: j \in \mathcal{J}\right\}$. Let $\left\{\tau_{k}: k \in \mathbb{N} \cap[0,|\mathcal{J}|]\right\}$ be the ordering of $\mathcal{T}$ such that $\tau_{k}<\tau_{k+1}$ for all $k<|\mathcal{J}|$. Note that $\mathcal{J}$ is an infinite set in most settings of interest. But it can happen that $|\mathcal{J}|<\infty$, for example when $\rho>1$ or when there are initial jobs larger than the supremum of the support of $\nu$. 
Lemma 2. (i) Almost surely, for all $k \in \mathbb{N}$ with $k \leq|\mathcal{J}|$,

$$
\left\langle\chi 1_{\left[F\left(\tau_{k}\right), \infty\right)}, \mathcal{Z}\left(\tau_{k}\right)\right\rangle=W\left(\tau_{k}\right) .
$$

(ii) Almost surely, for all $k<|\mathcal{J}|$ and $t \in\left(\tau_{k}, \tau_{k+1}\right]$,

$$
\left\langle\chi 1_{\left[F\left(\tau_{k}\right), \infty\right)}, \mathcal{Z}(t)\right\rangle=W\left(\tau_{k}\right)-F\left(\tau_{k}\right)+\sum_{j=E\left(\tau_{k}\right)+1}^{E(t)} w_{j} 1_{\left\{w_{j} \geq F\left(\tau_{k}\right)\right\}}
$$

Moreover, if $k=|\mathcal{J}|<\infty$ and $t \in\left(\tau_{k}, \infty\right)$, then (6) holds as well.

Proof. (i) Fix $k \in \mathbb{N} \cap[0,|\mathcal{J}|]$. By definition, $\tau_{k}=\gamma_{n\left(j_{0}\right)}$ for exactly one $j_{0} \in$ $\mathcal{J}$, and $F\left(\tau_{k}\right)=F\left(\gamma_{n\left(j_{0}\right)}\right)=w_{j_{0}}$. Consider any $j \in J\left(\tau_{k}\right)$. If $\gamma_{n(j)}<\tau_{k}$, then (2) implies that $\gamma_{n(j)}+y_{n(j)} \leq \gamma_{n\left(j_{0}\right)}$, and so $w_{j}\left(\tau_{k}\right)=0$. On the other hand, if $\gamma_{n(j)} \geq \tau_{k}$, then $w_{j}\left(\tau_{k}\right)=w_{j}$ and (2) implies that $j \in J\left(\gamma_{n\left(j_{0}\right)}\right) \backslash J_{n\left(j_{0}\right)-1}$. In particular, $w_{j} \geq y_{n\left(j_{0}\right)}=w_{j_{0}}$. So at time $\tau_{k}$, all arrived jobs have residual service time either equal to zero or bounded below by $F\left(\tau_{k}\right)$. This implies that the first right-hand term in

$$
W\left(\tau_{k}\right)=\left\langle\chi 1_{\left[0, F\left(\tau_{k}\right)\right)}, \mathcal{Z}\left(\tau_{k}\right)\right\rangle+\left\langle\chi 1_{\left[F\left(\tau_{k}\right), \infty\right)}, \mathcal{Z}\left(\tau_{k}\right)\right\rangle
$$

equals zero and proves (5).

(ii) To prove ([6), fix $k<|\mathcal{J}|$ and $t \in\left(\tau_{k}, \tau_{k+1}\right]$. Then

$$
\left\langle\chi 1_{\left[F\left(\tau_{k}\right), \infty\right)}, \mathcal{Z}(t)\right\rangle=\sum_{j \in J(t)} w_{j}(t) 1_{\left\{w_{j}(t) \geq F\left(\tau_{k}\right)\right\}} .
$$

Observe that $w_{j_{0}}(t)<w_{j_{0}}=F\left(\tau_{k}\right)$, because $t>\gamma_{n\left(j_{0}\right)}$ and $\gamma_{n\left(j_{0}\right)} \in \mathcal{T}$. So

$$
w_{j_{0}}(t) 1_{\left\{w_{j_{0}}(t) \geq F\left(\tau_{k}\right)\right\}}=0,
$$

while

$$
w_{j_{0}}\left(\tau_{k}\right) 1_{\left\{w_{j_{0}}\left(\tau_{k}\right) \geq F\left(\tau_{k}\right)\right\}}=F\left(\tau_{k}\right) .
$$

Now consider $j \in J(t) \backslash j_{0}$. We will show that

$$
w_{j}(t) 1_{\left\{w_{j}(t) \geq F\left(\tau_{k}\right)\right\}}=w_{j}\left(\tau_{k}\right) 1_{\left\{w_{j}\left(\tau_{k}\right) \geq F\left(\tau_{k}\right)\right\}} .
$$

Case one: If $\gamma_{n(j)}<\tau_{k}$, then $w_{j}\left(\tau_{k}\right)=0$ by (2) . Since $w_{j}(\cdot)$ is nonincreasing and $t>\tau_{k}, w_{j}(t)=0$ as well and so (10) holds.

Case two: If $\gamma_{n(j)} \in\left(\tau_{k}, \tau_{k+1}\right)$, then $j \notin \mathcal{J}$ and so $w_{j}<F\left(\gamma_{n(j)}\right)$. Since $F(\cdot)$ is right-continuous, piecewise constant, and can only jump at 
(a subset of) times $\tau \in \mathcal{T}$, this implies $w_{j}<F\left(\tau_{k}\right)$. Then $1_{\left\{w_{j}(t) \geq F\left(\tau_{k}\right)\right\}}=$ $1_{\left\{w_{j}\left(\tau_{k}\right) \geq F\left(\tau_{k}\right)\right\}}=0$ and equation (10) holds.

Case three: If $\gamma_{n(j)} \geq \tau_{k+1}$, then $\gamma_{n(j)} \geq t$ and so $s_{j}(t)=0$, that is, $w_{j}(t)=w_{j}\left(\tau_{k}\right)$ and equation (10) holds.

This proves (10). Combining (17) with (8) and (10) and noting that if $j>E\left(\tau_{k}\right)$ then $w_{j}\left(\tau_{k}\right)=w_{j}$, we obtain

$$
\left\langle\chi 1_{\left[F\left(\tau_{k}\right), \infty\right)}, \mathcal{Z}(t)\right\rangle=\sum_{j \in J\left(\tau_{k}\right) \backslash j_{0}} w_{j}\left(\tau_{k}\right) 1_{\left\{w_{j}\left(\tau_{k}\right) \geq F\left(\tau_{k}\right)\right\}}+\sum_{j=E\left(\tau_{k}\right)+1}^{E(t)} w_{j} 1_{\left\{w_{j} \geq F\left(\tau_{k}\right)\right\}} .
$$

By (9), including the $j_{0}$-term in the first sum is compensated by subtracting $F\left(\tau_{k}\right)$, which yields

$$
\left\langle\chi 1_{\left[F\left(\tau_{k}\right), \infty\right)}, \mathcal{Z}(t)\right\rangle=\left\langle\chi 1_{\left[F\left(\tau_{k}\right), \infty\right)}, \mathcal{Z}\left(\tau_{k}\right)\right\rangle-F\left(\tau_{k}\right)+\sum_{j=E\left(\tau_{k}\right)+1}^{E(t)} w_{j} 1_{\left\{w_{j} \geq F\left(\tau_{k}\right)\right\}}
$$

Applying (5) proves (6).

It remains to show (6) when $k=|\mathcal{J}|<\infty$ and $t \in\left(\tau_{k}, \infty\right)$. The argument is identical except for Cases two and three in the proof of (10). Case two becomes $\gamma_{n(j)} \in\left(\tau_{k}, \infty\right)$. Then $j \notin \mathcal{J}$ and $w_{j}<F\left(\gamma_{n(j)}\right)$. Since $F(\cdot)$ is constant on $\left[\tau_{k}, \infty\right), w_{j}<F\left(\tau_{k}\right)$ and (10) follows as before. Case three becomes $\gamma_{n(j)}=\infty$. Then $w_{j}\left(\tau_{k}\right)=w_{j}(t)=w_{j}$, which implies (10). The remainder of the proof follows from (7)-(10) as before.

We need a similar result for SRPT, proved in the next two lemmas. For each $j \in J$, the SRPT start time of job $j$ is defined $\eta_{j}=\inf \left\{t \geq 0: \dot{v}_{j}(t)=\right.$ $-1\}$. Let

$$
\mathcal{J}_{p}=\left\{j \in J: \eta_{j}<\infty \text { and } w_{j}=F_{p}\left(\eta_{j}\right)\right\},
$$

let $\mathcal{T}_{p}=\left\{\eta_{j}: j \in \mathcal{J}_{p}\right\}$, and let $\left\{\sigma_{k}: k \in \mathbb{N} \cap\left[0,\left|\mathcal{J}_{p}\right|\right]\right\}$ be the ordering of $\mathcal{T}_{p}$ such that $\sigma_{k}<\sigma_{k+1}$ for all $k<\left|\mathcal{J}_{p}\right|$.

Lemma 3. Almost surely, (i) if $t \in \mathcal{T}_{p}$, then $C_{p}(t)=F_{p}(t)$, and (ii) if $C_{p}(t)=F_{p}(t)>0$, then $t \in \mathcal{T}_{p}$.

Proof. Fix $t \in \mathcal{T}_{p}$, so $t=\eta_{j}$ for some $j \in \mathcal{J}_{p}$. Then $w_{j}=F_{p}\left(\eta_{j}\right)$. Evidently $v_{j}(t)=w_{j}>0$, so $\mathcal{Z}_{p}(t) \neq \mathbf{0}$, and therefore $C_{p}(t)=L_{p}(t)$. Now suppose $\left\langle 1_{\left[0, w_{j}\right)}, \mathcal{Z}_{p}(t)\right\rangle>0$. Then there is at least one job in the queue at time $t$ with residual service time $y \in\left(0, w_{j}\right)$, contradicting the assumption that SRPT starts job $j$ at $t$. This shows that $C_{p}(t) \geq F_{p}(t)$. Since $F_{p}(t) \geq C_{p}(t)$ by definition, this establishes (i). 
For (ii), fix $t$ such that $C_{p}(t)=F_{p}(t)>0$. By [4] Lemma 5.3(i), $\left\langle 1_{\left[0, C_{p}(t)\right)}, \mathcal{Z}_{p}(t)\right\rangle=0$ almost surely. Additionally, noting differences in the indexing of jobs, 4 Lemma 5.3(ii) implies that for all $j \in J$ such that $w_{j}>F_{p}(t), v_{j}(s)=w_{j}$ for all $s \in[0, t]$. Since $C_{p}(t)>0, \mathcal{Z}_{p}(t) \neq \mathbf{0}$ and so $\left\langle 1_{\left[C_{p}(t), \infty\right)}, \mathcal{Z}_{p}(t)\right\rangle>0$. Thus the set $\left\{j \in J(t): C_{p}(t)=v_{j}(t)=w_{j}\right\}$ is nonempty. Let $j^{*}$ be the smallest element of this set. Almost surely, there exists $\epsilon \in\left(0, C_{p}(t)\right)$ such that $E(t+\epsilon)=E(t)$, that is, no new jobs arrive during $(t, t+\epsilon]$. Then $\frac{d}{d s} v_{j^{*}}(s)=0$ on $[0, t)$ and $\frac{d}{d s} v_{j^{*}}(s)=-1$ on $(t, t+\epsilon)$, so $\eta_{j^{*}}=\inf \left\{s \geq 0: \dot{v}_{j^{*}}(s)=-1\right\}=t$. Thus $w_{j^{*}}=F_{p}(t)=F_{p}\left(\eta_{j^{*}}\right)$ and hence $j^{*} \in \mathcal{J}_{p}$ and $t \in \mathcal{T}_{p}$.

Lemma 4. (i) Almost surely, for all $k \in \mathbb{N}$ with $k \leq\left|\mathcal{J}_{p}\right|$,

$$
\left\langle\chi 1_{\left[F_{p}\left(\sigma_{k}\right), \infty\right)}, \mathcal{Z}_{p}\left(\sigma_{k}\right)\right\rangle=W\left(\sigma_{k}\right) .
$$

(ii) Almost surely, for all $k<\left|\mathcal{J}_{p}\right|$ and $t \in\left(\sigma_{k}, \sigma_{k+1}\right]$,

$$
\left\langle\chi 1_{\left[F_{p}\left(\sigma_{k}\right), \infty\right)}, \mathcal{Z}_{p}(t)\right\rangle=W\left(\sigma_{k}\right)-F_{p}\left(\sigma_{k}\right)+\sum_{j=E\left(\sigma_{k}\right)+1}^{E(t)} w_{j} 1_{\left\{w_{j} \geq F_{p}\left(\sigma_{k}\right)\right\}} .
$$

Moreover, if $k=\left|\mathcal{J}_{p}\right|<\infty$ and $t \in\left(\sigma_{k}, \infty\right)$, then (12) holds as well.

Proof. (i) Fix $k \in \mathbb{N} \cap\left[0,\left|\mathcal{J}_{p}\right|\right]$. By [4] Lemma $5.3(\mathrm{i}),\left\langle 1_{\left[0, C_{p}(t)\right)}, \mathcal{Z}_{p}(t)\right\rangle=0$ for all $t$ almost surely. Since $\sigma_{k} \in \mathcal{T}_{p}, F_{p}\left(\sigma_{k}\right)=C_{p}\left(\sigma_{k}\right)$ by Lemma 3, and it follows that $\left\langle 1_{\left[0, F_{p}\left(\sigma_{k}\right)\right)}, \mathcal{Z}_{p}\left(\sigma_{k}\right)\right\rangle=0$. This implies that the first right-hand term in

$$
W\left(\sigma_{k}\right)=\left\langle\chi 1_{\left[0, F_{p}\left(\sigma_{k}\right)\right)}, \mathcal{Z}_{p}\left(\sigma_{k}\right)\right\rangle+\left\langle\chi 1_{\left[F_{p}\left(\sigma_{k}\right), \infty\right)}, \mathcal{Z}_{p}\left(\sigma_{k}\right)\right\rangle
$$

equals zero and proves (11).

(ii) To prove (12), fix $k<\left|\mathcal{J}_{p}\right|$ and fix $t \in\left(\sigma_{k}, \sigma_{k+1}\right]$. Then

$$
\left\langle\chi 1_{\left[F_{p}\left(\sigma_{k}\right), \infty\right)}, \mathcal{Z}_{p}(t)\right\rangle=\sum_{j \in J(t)} v_{j}(t) 1_{\left\{v_{j}(t) \geq F_{p}\left(\sigma_{k}\right)\right\}} .
$$

Let $j_{0} \in \mathcal{J}_{p}$ be the job started at $\sigma_{k}$. Then $w_{j_{0}}=F_{p}\left(\sigma_{k}\right)$ and $v_{j_{0}}(t)<w_{j_{0}}$ since $t>\sigma_{k}=\eta_{j_{0}}$. Thus,

$$
v_{j_{0}}(t) 1_{\left\{v_{j_{0}}(t) \geq F_{p}\left(\sigma_{k}\right)\right\}}=0,
$$

while

$$
v_{j_{0}}\left(\sigma_{k}\right) 1_{\left\{v_{j_{0}}\left(\sigma_{k}\right) \geq F_{p}\left(\sigma_{k}\right)\right\}}=F_{p}\left(\sigma_{k}\right) .
$$


Now consider $j \in J(t) \backslash j_{0}$. We will show that

$$
v_{j}(t) 1_{\left\{v_{j}(t) \geq F_{p}\left(\sigma_{k}\right)\right\}}=v_{j}\left(\sigma_{k}\right) 1_{\left\{v_{j}\left(\sigma_{k}\right) \geq F_{p}\left(\sigma_{k}\right)\right\}} .
$$

Case one: If $\eta_{j}<\sigma_{k}$, then $\dot{v}_{j}(t)=-1$ on $\left(\eta_{j}, \eta_{j}+\epsilon\right)$ for some $\epsilon>0$, so $v_{j}\left(\sigma_{k}\right)<w_{j} \leq F_{p}\left(\eta_{j}\right) \leq F_{p}\left(\sigma_{k}\right)$. Since $v_{j}(\cdot)$ is nonincreasing and $t>\sigma_{k}$, we have $1_{\left\{v_{j}(t) \geq F_{p}\left(\sigma_{k}\right)\right\}}=1_{\left\{v_{j}\left(\sigma_{k}\right) \geq F_{p}\left(\sigma_{k}\right)\right\}}=0$, and (16) holds.

Case two: If $\eta_{j} \in\left(\sigma_{k}, \sigma_{k+1}\right)$, then $j \notin \mathcal{J}_{p}$ and so $w_{j}<F_{p}\left(\eta_{j}\right)$. Since $F_{p}(\cdot)$ is constant on $\left[\sigma_{k}, \sigma_{k+1}\right)$, this implies $v_{j}(t) \leq v_{j}\left(\sigma_{k}\right) \leq w_{j}<F_{p}\left(\sigma_{k}\right)$. Then $1_{\left\{v_{j}(t) \geq F_{p}\left(\sigma_{k}\right)\right\}}=1_{\left\{v_{j}\left(\sigma_{k}\right) \geq F_{p}\left(\sigma_{k}\right)\right\}}=0$ and (16) holds.

Case three: If $\eta_{j} \geq \sigma_{k+1}$, then $\eta_{j} \geq t$ and so $v_{j}(t)=v_{j}\left(\sigma_{k}\right)$ and (16) holds.

This proves (16). Combining (13) with (14) and (16) and noting that if $j>E\left(\sigma_{k}\right)$ then $v_{j}\left(\sigma_{k}\right)=w_{j}$, we obtain

$\left\langle\chi 1_{\left[F_{p}\left(\sigma_{k}\right), \infty\right)}, \mathcal{Z}_{p}(t)\right\rangle=\sum_{j \in J\left(\sigma_{k}\right) \backslash j 0} v_{j}\left(\sigma_{k}\right) 1_{\left\{v_{j}\left(\sigma_{k}\right) \geq F_{p}\left(\sigma_{k}\right)\right\}}+\sum_{j=E\left(\sigma_{k}\right)+1}^{E(t)} w_{j} 1_{\left\{w_{j} \geq F_{p}\left(\sigma_{k}\right)\right\}}$.

By (15), including the $j_{0}$-term in the first sum is compensated by subtracting $F_{p}\left(\sigma_{k}\right)$, which yields

$\left\langle\chi 1_{\left[F_{p}\left(\sigma_{k}\right), \infty\right)}, \mathcal{Z}_{p}(t)\right\rangle=\left\langle\chi 1_{\left[F_{p}\left(\sigma_{k}\right), \infty\right)}, \mathcal{Z}_{p}\left(\sigma_{k}\right)\right\rangle-F_{p}\left(\sigma_{k}\right)+\sum_{j=E\left(\sigma_{k}\right)+1}^{E(t)} w_{j} 1_{\left\{w_{j} \geq F_{p}\left(\sigma_{k}\right)\right\}}$.

Applying (11) proves (12).

It remains to show (12) when $k=\left|\mathcal{J}_{p}\right|<\infty$ and $t \in\left(\sigma_{k}, \infty\right)$. The argument is identical except for Cases two and three in the proof of (16). Case two becomes $\eta_{j} \in\left(\sigma_{k}, \infty\right)$. Then $j \notin \mathcal{J}_{p}$ and $w_{j}<F_{p}\left(\eta_{j}\right)$. Since $F_{p}(\cdot)$ is constant on $\left[\sigma_{k}, \infty\right), w_{j}<F_{p}\left(\sigma_{k}\right)$ and (16) follows as before. Case three becomes $\eta_{j}=\infty$. Then $v_{j}\left(\sigma_{k}\right)=v_{j}(t)=w_{j}$, which implies (16). The remainder of the proof follows from (13)-(16) as before.

We now show that SJF and SRPT have the same start times of frontier jobs, and coinciding frontiers at those times. This is the principal technical result of the paper.

Lemma 5. Almost surely, $\mathcal{T}=\mathcal{T}_{p}$ and $F(\tau)=F_{p}(\tau)$ for all $\tau \in \mathcal{T}$.

Proof. Clearly, $\sigma_{1}=\inf \left\{t \geq 0: Z_{0}+E(t) \geq 1\right\}=\gamma_{1}$. For the SJF queue, $w_{j_{1}}=y_{1}=F\left(\gamma_{1}\right)$, so $j_{1} \in \mathcal{J}$ and thus $\gamma_{1} \in \mathcal{T}$, implying $\tau_{1}=\gamma_{1}$. Conclude that $\tau_{1}=\sigma_{1}$. Moreover, $F\left(\tau_{1}\right)=\min \left\{w_{j}: j \in J\left(\tau_{1}\right)\right\}=F_{p}\left(\tau_{1}\right)$. Now fix 
$k<|\mathcal{J}| \wedge\left|\mathcal{J}_{p}\right|$ and assume that $\tau_{l}=\sigma_{l}$ and $F\left(\tau_{l}\right)=F_{p}\left(\tau_{l}\right)$ for all $l=1, \ldots, k$. We will show that $\tau_{k+1}=\sigma_{k+1}$ and $F\left(\tau_{k+1}\right)=F_{p}\left(\tau_{k+1}\right)$.

Note first that by definition of the start times, $\mathcal{Z}\left(\tau_{k+1}\right) \neq \mathbf{0}$ and $\mathcal{Z}_{p}\left(\sigma_{k+1}\right) \neq \mathbf{0}$. Since both queues have the same workload process $W(\cdot)$, this implies that $W\left(\tau_{k+1}\right) \wedge W\left(\sigma_{k+1}\right)>0$, and so $\mathcal{Z}\left(\sigma_{k+1}\right) \neq \mathbf{0}$ and $\mathcal{Z}_{p}\left(\tau_{k+1}\right) \neq \mathbf{0}$. We first show that $\tau_{k+1}=\sigma_{k+1}$.

Suppose that $\sigma_{k+1}>\tau_{k+1}$. Then $\tau_{k+1} \in\left(\sigma_{k}, \sigma_{k+1}\right)$, so by Lemma 4 and the induction hypothesis,

$$
\left\langle\chi 1_{\left[F_{p}\left(\sigma_{k}\right), \infty\right)}, \mathcal{Z}_{p}\left(\tau_{k+1}\right)\right\rangle=W\left(\tau_{k}\right)-F\left(\tau_{k}\right)+\sum_{j=E\left(\tau_{k}\right)+1}^{E\left(\tau_{k+1}\right)} w_{j} 1_{\left\{w_{j} \geq F\left(\tau_{k}\right)\right\}} .
$$

Using $t=\tau_{k+1}$ in (6) of Lemma 2, rewrite the right side of the display above to obtain

$$
\left\langle\chi 1_{\left[F_{p}\left(\sigma_{k}\right), \infty\right)}, \mathcal{Z}_{p}\left(\tau_{k+1}\right)\right\rangle=\left\langle\chi 1_{\left[F\left(\tau_{k}\right), \infty\right)}, \mathcal{Z}\left(\tau_{k+1}\right)\right\rangle .
$$

Since $F\left(\tau_{k}\right) \leq F\left(\tau_{k+1}\right)$, the right side is bounded below by $\left\langle\chi 1_{\left[F\left(\tau_{k+1}\right), \infty\right)}, \mathcal{Z}\left(\tau_{k+1}\right)\right\rangle$, and bounded above by $W\left(\tau_{k+1}\right)$, both of which are equal by (5) of Lemma 2. Thus,

$$
\left\langle\chi 1_{\left[F_{p}\left(\sigma_{k}\right), \infty\right)}, \mathcal{Z}_{p}\left(\tau_{k+1}\right)\right\rangle=W\left(\tau_{k+1}\right),
$$

which implies that

$$
\left\langle 1_{\left[0, F_{p}\left(\sigma_{k}\right)\right)}, \mathcal{Z}_{p}\left(\tau_{k+1}\right)\right\rangle=\left\langle\chi 1_{\left[0, F_{p}\left(\sigma_{k}\right)\right)}, \mathcal{Z}_{p}\left(\tau_{k+1}\right)\right\rangle=0 .
$$

Since $\mathcal{Z}_{p}\left(\tau_{k+1}\right) \neq \mathbf{0}$, (17) implies that $C_{p}\left(\tau_{k+1}\right) \geq F_{p}\left(\sigma_{k}\right)$. But $F_{p}(\cdot)$ is constant on $\left[\sigma_{k}, \sigma_{k+1}\right)$, so $C_{p}\left(\tau_{k+1}\right) \geq F_{p}\left(\tau_{k+1}\right)$, which implies by Lemma 3 that $\tau_{k+1} \in \mathcal{T}_{p}$, a contradiction.

Now suppose that $\sigma_{k+1}<\tau_{k+1}$. We proceed analogously to the above argument, with the roles of $\tau_{k+1}, \sigma_{k+1}$ and Lemmas 2 and 4 reversed. We have $\sigma_{k+1} \in\left(\tau_{k}, \tau_{k+1}\right)$, so by Lemma 2 and the induction hypothesis,

$$
\left\langle\chi 1_{\left[F\left(\tau_{k}\right), \infty\right)}, \mathcal{Z}\left(\sigma_{k+1}\right)\right\rangle=W\left(\sigma_{k}\right)-F_{p}\left(\sigma_{k}\right)+\sum_{j=E\left(\sigma_{k}\right)+1}^{E\left(\sigma_{k+1}\right)} w_{j} 1_{\left\{w_{j} \geq F_{p}\left(\sigma_{k}\right)\right\}} .
$$

Using $t=\sigma_{k+1}$ in (12) of Lemma 4, rewrite the right side of the display above to obtain

$$
\left\langle\chi 1_{\left[F\left(\tau_{k}\right), \infty\right)}, \mathcal{Z}\left(\sigma_{k+1}\right)\right\rangle=\left\langle\chi 1_{\left[F_{p}\left(\sigma_{k}\right), \infty\right)}, \mathcal{Z}_{p}\left(\sigma_{k+1}\right)\right\rangle
$$


Since $F_{p}\left(\sigma_{k}\right) \leq F_{p}\left(\sigma_{k+1}\right)$, the right side is bounded below by $\left\langle\chi 1_{\left[F_{p}\left(\sigma_{k+1}\right), \infty\right)}, \mathcal{Z}_{p}\left(\sigma_{k+1}\right)\right\rangle$, and bounded above by $W\left(\sigma_{k+1}\right)$, both of which are equal by (11) of Lemma 4. Thus,

$$
\left\langle\chi 1_{\left[F\left(\tau_{k}\right), \infty\right)}, \mathcal{Z}\left(\sigma_{k+1}\right)\right\rangle=W\left(\sigma_{k+1}\right),
$$

which, since $F(\cdot)$ is constant on $\left[\tau_{k}, \tau_{k+1}\right)$, implies that

$$
\left\langle\chi 1_{\left[0, F\left(\sigma_{k+1}\right)\right)}, \mathcal{Z}\left(\sigma_{k+1}\right)\right\rangle=0 .
$$

Since $\mathcal{Z}\left(\sigma_{k+1}\right) \neq \mathbf{0},(18)$ implies that the set $I=\left\{j \in J\left(\sigma_{k+1}\right): w_{j}\left(\sigma_{k+1}\right) \geq\right.$ $\left.F\left(\sigma_{k+1}\right)\right\}$ is nonempty. Let $j^{*}=\operatorname{argmin}\left\{\gamma_{n(j)}: j \in I\right\}$ be the job in $I$ with the earliest start time. Then by (18), $w_{j_{n\left(j^{*}\right)-1}}\left(\sigma_{k+1}\right)=0$, which implies that $\gamma_{n\left(j^{*}\right)-1}+y_{n\left(j^{*}\right)-1} \leq \sigma_{k+1}$ and $Z_{0}+E\left(\sigma_{k+1}\right) \geq n\left(j^{*}\right)$. Conclude from (2) that $\gamma_{n\left(j^{*}\right)}=\sigma_{k+1}$. Moreover, the definition of $F(\cdot)$ implies that $w_{j^{*}}=F\left(\sigma_{k+1}\right)$, and so $\sigma_{k+1} \in \mathcal{T}$, a contradiction.

The previous two arguments imply that $\tau_{k+1}=\sigma_{k+1}$. We now show that $F\left(\tau_{k+1}\right)=F_{p}\left(\tau_{k+1}\right)$. Suppose that $F_{p}\left(\tau_{k+1}\right)>F\left(\tau_{k+1}\right)$ so that the interval $\left[F\left(\tau_{k+1}\right), F_{p}\left(\tau_{k+1}\right)\right)$ is nonempty. By definition of $\mathcal{T}$, there is a job $j_{*} \in \mathcal{J}$ such that $\gamma_{n\left(j_{*}\right)}=\tau_{k+1}$ and $w_{j_{*}}=F\left(\tau_{k+1}\right)$. Then $w_{j_{*}}\left(\tau_{k+1}\right)=w_{j_{*}}$ and so

$$
\left\langle\chi 1_{\left[F\left(\tau_{k+1}\right), F_{p}\left(\tau_{k+1}\right)\right)}, \mathcal{Z}\left(\tau_{k+1}\right)\right\rangle>0 .
$$

Note that for $j \in J\left(\tau_{k+1}\right), w_{j} \geq F_{p}\left(\tau_{k+1}\right)$ implies that $w_{j}>F\left(\tau_{k+1}\right)$ and so $w_{j}\left(\tau_{k+1}\right)=w_{j} \geq F_{p}\left(\tau_{k+1}\right)$. Conversely, $w_{j}\left(\tau_{k+1}\right) \geq F_{p}\left(\tau_{k+1}\right)$ clearly implies $w_{j} \geq w_{j}\left(\tau_{k+1}\right) \geq F_{p}\left(\tau_{k+1}\right)$. So

$$
w_{j}\left(\tau_{k+1}\right) 1_{\left\{w_{j}\left(\tau_{k+1}\right) \geq F_{p}\left(\tau_{k+1}\right)\right\}}=w_{j} 1_{\left\{w_{j} \geq F_{p}\left(\tau_{k+1}\right)\right\}} .
$$

Similarly, $w_{j} \geq F_{p}\left(\tau_{k+1}\right)$ implies $w_{j}>F\left(\tau_{k+1}\right) \geq F\left(\tau_{k}\right)=F_{p}\left(\tau_{k}\right)$, and so $v_{j}\left(\tau_{k+1}\right)=w_{j} \geq F_{p}\left(\tau_{k+1}\right)$. Conversely, $v_{j}\left(\tau_{k+1}\right) \geq F_{p}\left(\tau_{k+1}\right)$ gives $w_{j} \geq$ $v_{j}\left(\tau_{k+1}\right) \geq F_{p}\left(\tau_{k+1}\right)$ and so

$$
v_{j}\left(\tau_{k+1}\right) 1_{\left\{v_{j}\left(\tau_{k+1}\right) \geq F_{p}\left(\tau_{k+1}\right)\right\}}=w_{j} 1_{\left\{w_{j} \geq F_{p}\left(\tau_{k+1}\right)\right\}} .
$$

Together, (20) and (21) imply that

$$
\sum_{j \in J\left(\tau_{k+1}\right)} w_{j}\left(\tau_{k+1}\right) 1_{\left\{w_{j}\left(\tau_{k+1}\right) \geq F_{p}\left(\tau_{k+1}\right)\right\}}=\sum_{j \in J\left(\tau_{k+1}\right)} v_{j}\left(\tau_{k+1}\right) 1_{\left\{v_{j}\left(\tau_{k+1}\right) \geq F_{p}\left(\tau_{k+1}\right)\right\}}
$$

and so

$$
\left\langle\chi 1_{\left[F_{p}\left(\tau_{k+1}\right), \infty\right)}, \mathcal{Z}\left(\tau_{k+1}\right)\right\rangle=\left\langle\chi 1_{\left[F_{p}\left(\tau_{k+1}\right), \infty\right)}, \mathcal{Z}_{p}\left(\tau_{k+1}\right)\right\rangle .
$$


By (22),

$$
\begin{array}{r}
\left\langle\chi 1_{\left[F\left(\tau_{k+1}\right), \infty\right)}, \mathcal{Z}\left(\tau_{k+1}\right)\right\rangle=\left\langle\chi 1_{\left[F\left(\tau_{k+1}\right), F_{p}\left(\tau_{k+1}\right)\right)}, \mathcal{Z}\left(\tau_{k+1}\right)\right\rangle \\
+\left\langle\chi 1_{\left[F_{p}\left(\tau_{k+1}\right), \infty\right)}, \mathcal{Z}\left(\tau_{k+1}\right)\right\rangle \\
=\left\langle\chi 1_{\left[F\left(\tau_{k+1}\right), F_{p}\left(\tau_{k+1}\right)\right)}, \mathcal{Z}\left(\tau_{k+1}\right)\right\rangle \\
+\left\langle\chi 1_{\left[F_{p}\left(\tau_{k+1}\right), \infty\right)}, \mathcal{Z}_{p}\left(\tau_{k+1}\right)\right\rangle
\end{array}
$$

But the left side and second term on the right side of (23) are both equal to $W\left(\tau_{k+1}\right)$ by Lemmas 2 and 4 . So the first term on the right side equals zero, contradicting (19). This argument is symmetric if the roles of $F\left(\tau_{k+1}\right)$ and $F_{p}\left(\tau_{k+1}\right)$ are reversed, and we conclude that $F\left(\tau_{k+1}\right)=F_{p}\left(\tau_{k+1}\right)$.

This completes the proof if $|\mathcal{J}|=\left|\mathcal{J}_{p}\right|$. But this is the only possibility, for if $|\mathcal{J}|>\left|\mathcal{J}_{p}\right|$, then for $k=\left|\mathcal{J}_{p}\right|$, there exists $\tau_{k+1} \in \mathcal{J}$ such that $\tau_{k+1} \in$ $\left(\sigma_{k}, \infty\right)$. This yields a contradiction by the argument in the paragraph leading to (17) (substituting $\infty$ for $\sigma_{k+1}$ there). A similar contradiction is implied by $|\mathcal{J}|<\left|\mathcal{J}_{p}\right|$.

Corollary 1. Almost surely, $F(\cdot) \equiv F_{p}(\cdot)$ and for all $t \geq 0$,

$$
\left\langle\chi 1_{[F(t), \infty)}, \mathcal{Z}(t)\right\rangle=\left\langle\chi 1_{[F(t), \infty)}, \mathcal{Z}_{p}(t)\right\rangle
$$

Proof. The first statement follows from Lemma 5 since both $F(\cdot)$ and $F_{p}(\cdot)$ are right-continuous and constant on the intervals $\left[\tau_{k}, \tau_{k+1}\right)$ for all $k<|\mathcal{J}|$ and $\left[\tau_{k}, \infty\right)$ for $k=|\mathcal{J}|$. The second statement follows from this fact as well, combined with the first statement, and Lemmas 2, 4, and 5.

Since the frontier processes are identical, the notation $F(t)$ will be used hereafter for the frontier process under either policy. We will use the fact that the state descriptors under either policy are identical above the frontier.

Corollary 2. Almost surely, for all measurable $f: \mathbb{R}_{+} \rightarrow \mathbb{R}_{+}$and all $t \geq 0$,

$$
\left\langle f 1_{[F(t), \infty)}, \mathcal{Z}(t)\right\rangle=\left\langle f 1_{[F(t), \infty)}, \mathcal{Z}_{p}(t)\right\rangle .
$$

Proof. Fix $t \geq 0$. Clearly for all $j \in J(t), w_{j}(t)>F(t)$ if and only if $w_{j}>F(t)$ and $w_{j}(t)=w_{j}$, and $v_{j}(t)>F(t)$ if and only if $w_{j}>F(t)$ and $v_{j}(t)=w_{j}$. So for all measurable $f$,

$$
\left\langle f 1_{(F(t), \infty)}, \mathcal{Z}(t)\right\rangle=\sum_{j \in J(t)} f\left(w_{j}\right) 1_{\left\{w_{j}>F(t)\right\}}=\left\langle f 1_{(F(t), \infty)}, \mathcal{Z}_{p}(t)\right\rangle .
$$

Using the special case $f=\chi$ and combining with Corollary 1 yields

$$
\left\langle 1_{\{F(t)\}}, \mathcal{Z}(t)\right\rangle=\left\langle 1_{\{F(t)\}}, \mathcal{Z}_{p}(t)\right\rangle .
$$

Combining (24) and (25) completes the proof. 
We will also need the following simple bound for the workload below the frontier in the SRPT queue. By the workload equation in (1),

$$
\begin{aligned}
W(s) & =W(t)-(V(t)-V(s))+t-s-(I(t)-I(s)) \\
& \leq W(t)-(V(t)-V(s))+t-s,
\end{aligned}
$$

almost surely for all $t \geq s \geq 0$, since the cumulative idle time process $I(\cdot)$ is nondecreasing. By (4), and by splitting the workload at times $s$ and $t$ into residual service times that are strictly below the frontier, and those that are at or above the frontier,

$$
\begin{aligned}
\left\langle\chi 1_{[0, F(s))},\right. & \left.\mathcal{Z}_{p}(s)\right\rangle+\left\langle\chi 1_{[F(s), \infty)}, \mathcal{Z}_{p}(s)\right\rangle \\
& \leq\left\langle\chi 1_{[0, F(t))}, \mathcal{Z}_{p}(t)\right\rangle+\left\langle\chi 1_{[F(t), \infty)}, \mathcal{Z}_{p}(t)\right\rangle-\sum_{j=E(s)+1}^{E(t)} w_{j}+t-s .
\end{aligned}
$$

Note that $v_{j}(u) \geq F(u)$ implies $v_{j}(u)=w_{j}$ for all $u \geq 0$ and $j \in J(u)$.

Thus,

$$
\begin{aligned}
& \left\langle\chi 1_{[0, F(s))}, \mathcal{Z}_{p}(s)\right\rangle+\sum_{j \in J(s)} w_{j} 1_{\left\{v_{j}(s) \geq F(s)\right\}} \\
& \leq\left\langle\chi 1_{[0, F(t))}, \mathcal{Z}_{p}(t)\right\rangle+\sum_{j \in J(t)} w_{j} 1_{\left\{v_{j}(t) \geq F(t)\right\}}-\sum_{j=E(s)+1}^{E(t)} w_{j}+t-s .
\end{aligned}
$$

Since $F(\cdot)$ is non-decreasing and residual service times $v_{j}(\cdot)$ are non-increasing, $w_{j} 1_{\left\{v_{j}(t) \geq F(t)\right\}} \leq w_{j} 1_{\left\{v_{j}(s) \geq F(s)\right\}}$ for all $j \in J(s)$. Also, $w_{j} 1_{\left\{v_{j}(t) \geq F(t)\right\}} \leq w_{j}$ for all $j=E(s)+1, \ldots, E(t)$. So

$$
\sum_{j \in J(t)} w_{j} 1_{\left\{v_{j}(t) \geq F(t)\right\}}-\sum_{j=E(s)+1}^{E(t)} w_{j}-\sum_{j \in J(s)} w_{j} 1_{\left\{v_{j}(s) \geq F(s)\right\}} \leq 0,
$$

and thus almost surely for all $t \geq s \geq 0$,

$$
\left\langle\chi 1_{[0, F(s))}, \mathcal{Z}_{p}(s)\right\rangle \leq\left\langle\chi 1_{[0, F(t))}, \mathcal{Z}_{p}(t)\right\rangle+t-s .
$$

\section{Invariance of fluid limit}

Now we shift our point of view from a single model to a sequence of models. After defining the sequence and making appropriate asymptotic assumptions, we will use the work of the previous section to show that the models have the same fluid limit under SJF and SRPT. 
Let $\mathcal{R}$ be a sequence of positive real numbers increasing to infinity. For each $r \in \mathcal{R}$, there is an associated stochastic model with initial condition $Z_{0}^{r}$ and $\left\{w_{j}^{r}: j=\ldots,-2,-1\right\}$, arrival process $E^{r}(\cdot)$ and service times $\left\{w_{j}^{r}: j=1,2, \ldots\right\}$. These primitives have parameters $\alpha^{r}, \nu^{r}$, and $\rho^{r}$, and are defined on a probability space $\left(\Omega^{r}, \mathcal{F}^{r}, \mathbf{P}^{r}\right)$. Then for each $r \in \mathcal{R}$, the stochastic primitives give rise to measure-valued state descriptors $\mathcal{Z}^{r}(\cdot)$ and $\mathcal{Z}_{p}^{r}(\cdot)$ under the SJF and SRPT policies respectively, as well as a common frontier process $F^{r}(\cdot)$. Define fluid-scaled versions of the following processes:

$$
\begin{aligned}
\bar{F}^{r}(t) & =F^{r}(r t), \\
\overline{\mathcal{V}}^{r}(t) & =\frac{1}{r} \mathcal{V}^{r}(r t), \\
\bar{V}^{r}(t) & =\frac{1}{r} V^{r}(r t), \\
\overline{\mathcal{Z}}^{r}(t) & =\frac{1}{r} \mathcal{Z}^{r}(r t), \\
\overline{\mathcal{Z}}_{p}^{r}(t) & =\frac{1}{r} \mathcal{Z}_{p}^{r}(r t), \\
\bar{W}^{r}(t) & =\frac{1}{r} W^{r}(r t) .
\end{aligned}
$$

Let $\alpha>0$ and $\nu \in \mathbf{M}$ be a probability measure that does not charge the origin, such that $\rho=\alpha\langle\chi, \nu\rangle \leq 1$. We impose the following asymptotic assumptions, as $r \rightarrow \infty$, on the sequence of models. For the sequence of exogenous arrival processes, assume that

$$
\frac{1}{r} E^{r}(r t) \Rightarrow \alpha(\cdot)
$$

where $\alpha(t)=\alpha t$ for all $t \geq 0$. For the sequence of service time distributions, assume that

$$
\nu^{r} \stackrel{w}{\longrightarrow} \nu \text { and } \quad\left\{\nu^{r}: r \in \mathcal{R}\right\} \quad \text { is uniformly integrable. }
$$

It follows from (29) that $\rho^{r} \rightarrow \rho$.

Additionally, assume that as $r \rightarrow \infty$,

$$
\left(\overline{\mathcal{Z}}^{r}(0),\left\langle\chi, \overline{\mathcal{Z}}^{r}(0)\right\rangle\right) \Rightarrow\left(\mathcal{Z}_{0},\left\langle\chi, \mathcal{Z}_{0}\right\rangle\right)
$$

where $\mathcal{Z}_{0} \in \mathbf{M}$ is a random measure satisfying

$$
\begin{aligned}
\left\langle\chi, \mathcal{Z}_{0}\right\rangle & <\infty \quad \text { a.s. } \\
\left\langle 1_{\{0\}}, \mathcal{Z}_{0}\right\rangle & =0 \quad \text { a.s. }
\end{aligned}
$$


It is shown in [4] that, under the assumptions (28)-(32) as $r \rightarrow \infty$, there is joint convergence

$$
\left(\overline{\mathcal{V}}^{r}(\cdot), \bar{V}^{r}(\cdot), \bar{W}^{r}(0), \overline{\mathcal{Z}}_{p}^{r}(\cdot)\right) \Rightarrow\left(\mathcal{V}^{*}(\cdot), V^{*}(\cdot), W^{*}(0), \mathcal{Z}^{*}(\cdot)\right) .
$$

Here, $\mathcal{V}^{*}(t)=\alpha t \nu$ for all $t \geq 0$, and $\mathcal{Z}^{*}(\cdot)$ is almost surely an SRPT fluid model solution for data $(\alpha, \nu)$ and initial condition $\mathcal{Z}^{*}(0)$, equal in distribution to $\mathcal{Z}_{0}$; see Theorem 5.16 in [4] as well as Section 2.2 in [4] for a definition of the fluid model solutions. Such fluid model solutions are analyzed in detail in [4, 5].

Within the proof of (33), it is assumed by invoking the Skorohod representation theorem that all random elements are defined on a common probability space $\left(\Omega^{*}, \mathcal{F}^{*}, \mathbf{P}^{*}\right)$ such that, almost surely as $r \rightarrow \infty$,

$$
\left(\tilde{\mathcal{V}}^{r}(\cdot), \tilde{V}^{r}(\cdot), \tilde{W}^{r}(0), \tilde{\mathcal{Z}}_{p}^{r}(\cdot)\right) \rightarrow\left(\mathcal{V}^{*}(\cdot), V^{*}(\cdot), W^{*}(0), \mathcal{Z}^{*}(\cdot)\right)
$$

uniformly on compact time intervals, where all objects with a tilde denote Skorohod representations; see (84) in [4]. As part of this proof, it is shown that almost surely,

$$
\lim _{r \rightarrow \infty}\left\langle\chi 1_{\left[0, \tilde{F}^{r}(t)\right)}, \tilde{\mathcal{Z}}_{p}^{r}(t)\right\rangle=0, \quad \text { for all } t \geq 0
$$

where $\tilde{F}^{r}(t)=\sup _{s \in[0, t]} \sup \left\{x \in \mathbb{R}_{+}:\left\langle 1_{[0, x)}, \tilde{\mathcal{Z}}_{p}^{r}(s)\right\rangle=0\right\}$ with $\sup \varnothing=0$. (See (96) in the proof of Lemma 5.23(i) in [4]. Note that since $\rho \leq 1,5.23(\mathrm{i})$ is the relevant statement. Also, see the beginning of Section 5.3 in [4]; a fixed $\omega$ is chosen from an event of probability one in $\Omega^{*}$. Since Lemma 5.23 is proved for this fixed $\omega$, (35) holds almost surely.) We will use this fact to establish a similar statement for the original process $\overline{\mathcal{Z}}_{p}^{r}(\cdot)$, but we need to upgrade the pointwise convergence to uniform convergence on compact time intervals $[0, T]$. This is shown in the proof of the following lemma.

Lemma 6. For all $T \in[0, \infty)$,

$$
\sup _{t \in[0, T]}\left\langle\chi 1_{\left[0, \bar{F}^{r}(t)\right)}, \overline{\mathcal{Z}}_{p}^{r}(t)\right\rangle \stackrel{\mathbf{P}}{\longrightarrow} 0, \quad \text { as } r \rightarrow \infty .
$$

Proof. Since the Skorohod representations in (34) are equal in distribution to the original processes, it suffices to show that, $\mathbf{P}^{*}$-almost surely,

$$
\lim _{r \rightarrow \infty} \sup _{t \in[0, T]}\left\langle\chi 1_{\left[0, \tilde{F}^{r}(t)\right)}, \tilde{\mathcal{Z}}_{p}^{r}(t)\right\rangle=0 .
$$


To that end, let $\mathcal{D} \subset \Omega^{*}$ be the event of probability one on which (35) holds, and for each $r \in \mathcal{R}$ define the event

$$
\begin{aligned}
\mathcal{E}^{r}=\left\{\left\langle\chi 1_{\left[0, \tilde{F}^{r}(s)\right)}, \tilde{\mathcal{Z}}_{p}^{r}(s)\right\rangle \leq\left\langle\chi 1_{\left[0, \tilde{F}^{r}(t)\right)}, \tilde{\mathcal{Z}}_{p}^{r}(t)\right\rangle+t-s\right. & \\
& \text { for all } 0 \leq s \leq t<\infty\} .
\end{aligned}
$$

Since the dynamic inequality (26) is true almost surely, it also holds under fluid scaling $\mathbf{P}^{r}$-almost surely for each $r \in \mathcal{R}$, and therefore $\mathbf{P}^{*}$-almost surely for each $\tilde{\mathcal{Z}}_{p}^{r}(\cdot), r \in \mathcal{R}$. Thus, $\mathbf{P}^{*}\left(\mathcal{D} \cap \bigcap_{r \in \mathcal{R}} \mathcal{E}^{r}\right)=1$.

Fix $T \in[0, \infty)$ and $\epsilon>0$. Let $0=a_{0}, a_{1}, \ldots, a_{k}=T$ be a partition of $[0, T]$ such that $\max _{j \leq k} a_{j}-a_{j-1} \leq \epsilon$. For $t \in[0, T]$, let $a_{t}=\min \left\{a_{j}: a_{j} \geq\right.$ $t\}$. Then on $\mathcal{D}$,

$$
\lim _{r \rightarrow \infty} \max _{j \leq k}\left\langle\chi 1_{\left[0, \tilde{F}^{r}\left(a_{j}\right)\right)}, \tilde{\mathcal{Z}}_{p}^{r}\left(a_{j}\right)\right\rangle=0
$$

and on $\bigcap_{r \in \mathcal{R}} \mathcal{E}^{r}$, for all $r \in \mathcal{R}$ and $t \in[0, T]$,

$$
\begin{aligned}
\left\langle\chi 1_{\left[0, \tilde{F}^{r}(t)\right)}, \tilde{\mathcal{Z}}_{p}^{r}(t)\right\rangle & \leq\left\langle\chi 1_{\left[0, \tilde{F}^{r}\left(a_{t}\right)\right)}, \tilde{\mathcal{Z}}_{p}^{r}\left(a_{t}\right)\right\rangle+a_{t}-t \\
& \leq \max _{j \leq k}\left\langle\chi 1_{\left[0, \tilde{F}^{r}\left(a_{j}\right)\right)}, \tilde{\mathcal{Z}}_{p}^{r}\left(a_{j}\right)\right\rangle+\epsilon .
\end{aligned}
$$

So on $\mathcal{D} \cap \bigcap_{r \in \mathcal{R}} \mathcal{E}^{r}$,

$$
\limsup _{r \rightarrow \infty} \sup _{t \in[0, T]}\left\langle\chi 1_{\left[0, \tilde{F}^{r}(t)\right)}, \tilde{\mathcal{Z}}_{p}^{r}(t)\right\rangle \leq \epsilon
$$

which proves (36).

Next, we establish a bound for the fluid scaled mass near the origin, which is valid under either policy.

Lemma 7. Let $T<\infty$. For each $\epsilon, \eta \in(0,1)$ there exists $\delta>0$ such that

$$
\liminf _{r \rightarrow \infty} \mathbf{P}^{r}\left(\sup _{t \in[0, T]}\left(\left\langle 1_{[0, \delta]}, \overline{\mathcal{Z}}^{r}(t)\right\rangle \vee\left\langle 1_{[0, \delta]}, \overline{\mathcal{Z}}_{p}^{r}(t)\right\rangle\right) \leq \epsilon\right) \geq 1-\eta .
$$

Proof. Fix $\epsilon, \eta \in(0,1)$. By Lemma 5.11 in [4], there exists $\delta_{1}>0$ such that

$$
\liminf _{r \rightarrow \infty} \mathbf{P}^{r}\left(\sup _{t \in[0, T]}\left\langle 1_{\left[0, \delta_{1}\right]}, \overline{\mathcal{Z}}_{p}^{r}(t)\right\rangle \leq \epsilon\right) \geq 1-\frac{\eta}{2} .
$$


By (32), there exists $\delta_{2}>0$ such that

$$
\mathbf{P}\left(\left\langle 1_{\left[0, \delta_{2}\right]}, \mathcal{Z}_{0}\right\rangle<\frac{\epsilon}{3}\right) \geq 1-\frac{\eta}{2} .
$$

Since $\nu$ puts no mass at the origin, there exists $\delta_{3}>0$ such that

$$
\alpha T\left\langle 1_{\left[0, \delta_{3}\right]}, \nu\right\rangle<\frac{\epsilon}{3} .
$$

Let $\delta=\min \left\{\delta_{1}, \delta_{2}, \delta_{3}\right\}$ and define the events

$$
\begin{aligned}
& \Omega_{1}^{r}=\left\{\sup _{t \in[0, T]}\left\langle 1_{[0, \delta]}, \overline{\mathcal{Z}}_{p}^{r}(t)\right\rangle \leq \epsilon\right\}, \\
& \Omega_{2}^{r}=\left\{\left\langle 1_{[0, \delta]}, \overline{\mathcal{Z}}^{r}(0)\right\rangle<\frac{\epsilon}{3}\right\}, \\
& \Omega_{3}^{r}=\left\{\left\langle 1_{[0, \delta]}, \overline{\mathcal{V}}^{r}(T)\right\rangle<\frac{\epsilon}{3}\right\} .
\end{aligned}
$$

Then $\liminf _{r \rightarrow \infty} \mathbf{P}^{r}\left(\Omega_{1}^{r}\right) \geq 1-\eta / 2$ since $\delta \leq \delta_{1}$. The set $\{\xi \in \mathbf{M}$ : $\left.\left\langle 1_{\left[0, \delta_{2}\right]}, \xi\right\rangle<\epsilon / 3\right\}$ is open in the weak topology. So by (30), (37), the Portmanteau theorem, and since $\delta \leq \delta_{2}, \liminf _{r \rightarrow \infty} \mathbf{P}^{r}\left(\Omega_{2}^{r}\right) \geq 1-\eta / 2$. Similarly, by (33), (38), the Portmanteau theorem, and since $\delta \leq \delta_{3}, \liminf _{r \rightarrow \infty} \mathbf{P}^{r}\left(\Omega_{3}^{r}\right)=$ 1. Hence,

$$
\liminf _{r \rightarrow \infty} \mathbf{P}^{r}\left(\Omega_{1}^{r} \cap \Omega_{2}^{r} \cap \Omega_{3}^{r}\right) \geq 1-\eta .
$$

Note that for all $t \geq 0$, at most one $j \in J(t)$ satisfies $0<w_{j}(t)<w_{j}$ by (2). So almost surely for all $t \geq 0, \sum_{j \in J(t)} 1_{(0, \delta]}\left(w_{j}(t)\right) 1_{(\delta, \infty)}\left(w_{j}\right) \leq 1$. Under fluid scaling, this implies

$$
\sup _{t \in[0, T]}\left\langle 1_{[0, \delta]}, \overline{\mathcal{Z}}^{r}(t)\right\rangle \leq\left\langle 1_{[0, \delta]}, \overline{\mathcal{Z}}^{r}(0)\right\rangle+\left\langle 1_{[0, \delta]}, \overline{\mathcal{V}}^{r}(T)\right\rangle+\frac{1}{r} .
$$

Then almost surely on $\Omega_{1}^{r} \cap \Omega_{2}^{r} \cap \Omega_{3}^{r}$,

$$
\sup _{t \in[0, T]}\left\langle 1_{[0, \delta]}, \overline{\mathcal{Z}}^{r}(t)\right\rangle \leq \frac{\epsilon}{3}+\frac{\epsilon}{3}+\frac{1}{r},
$$

which is bounded above by $\epsilon$ for sufficiently large $r$.

We are now ready to prove that the fluid scaled state descriptors under SJF and SRPT converge together.

Theorem 2. For all $T<\infty$,

$$
\sup _{t \in[0, T]} \mathbf{d}\left[\overline{\mathcal{Z}}^{r}(t), \overline{\mathcal{Z}}_{p}^{r}(t)\right] \stackrel{\mathbf{P}}{\longrightarrow} 0, \quad \text { as } r \rightarrow \infty .
$$


Proof. Fix $T<\infty$ and $\epsilon, \eta>0$. By Lemma 7, there exists $\delta>0$ such that the events

$$
\Omega_{1}^{r}=\left\{\sup _{t \in[0, T]}\left(\left\langle 1_{[0, \delta]}, \overline{\mathcal{Z}}^{r}(t)\right\rangle \vee\left\langle 1_{[0, \delta]}, \overline{\mathcal{Z}}_{p}^{r}(t)\right\rangle\right) \leq \frac{\epsilon}{2}\right\}
$$

satisfy $\liminf \operatorname{in}_{r \rightarrow \infty} \mathbf{P}^{r}\left(\Omega_{1}^{r}\right) \geq 1-\eta / 2$. By Lemma 6 , the events

$$
\Omega_{2}^{r}=\left\{\sup _{t \in[0, T]}\left\langle\chi 1_{\left[0, \bar{F}^{r}(t)\right)}, \overline{\mathcal{Z}}_{p}^{r}(t)\right\rangle \leq \frac{\delta \epsilon}{2}\right\}
$$

satisfy $\liminf \operatorname{in}_{r \rightarrow \infty} \mathbf{P}^{r}\left(\Omega_{2}^{r}\right) \geq 1-\eta / 2$, and so $\liminf _{r \rightarrow \infty} \mathbf{P}^{r}\left(\Omega_{1}^{r} \cap \Omega_{2}^{r}\right) \geq 1-\eta$.

Fix $t \in[0, T]$ and let $B \subset \mathbb{R}_{+}$be closed. By intersecting $B$ with $[0, \delta]$, $\left(\delta, \bar{F}^{r}(t)\right)$, and $\left[\bar{F}^{r}(t), \infty\right)$, and by Markov's inequality,

$$
\left\langle 1_{B}, \overline{\mathcal{Z}}^{r}(t)\right\rangle \leq\left\langle 1_{[0, \delta]}, \overline{\mathcal{Z}}^{r}(t)\right\rangle+\frac{1}{\delta}\left\langle\chi 1_{\left[0, \bar{F}^{r}(t)\right)}, \overline{\mathcal{Z}}^{r}(t)\right\rangle+\left\langle 1_{\left.\left[\bar{F}^{r}(t)\right), \infty\right) \cap B}, \overline{\mathcal{Z}}^{r}(t)\right\rangle .
$$

We can replace $\overline{\mathcal{Z}}^{r}(t)$ by $\overline{\mathcal{Z}}_{p}^{r}(t)$ almost surely in the last term by Corollary 2, and in the next to last term by Corollary 1, since

$$
\left\langle\chi 1_{\left[0, \bar{F}^{r}(t)\right)}, \overline{\mathcal{Z}}^{r}(t)\right\rangle=\bar{W}^{r}(t)-\left\langle\chi 1_{\left[\bar{F}^{r}(t), \infty\right)}, \overline{\mathcal{Z}}^{r}(t)\right\rangle .
$$

Then use $\left.\left[\bar{F}^{r}(t)\right), \infty\right) \cap B \subset B^{\epsilon}$ to get, almost surely on $\Omega_{1}^{r} \cap \Omega_{2}^{r}$,

$$
\left\langle 1_{B}, \overline{\mathcal{Z}}^{r}(t)\right\rangle \leq \frac{\epsilon}{2}+\frac{\epsilon}{2}+\left\langle 1_{B^{\epsilon}}, \overline{\mathcal{Z}}_{p}^{r}(t)\right\rangle .
$$

The same argument yields, almost surely on $\Omega_{1}^{r} \cap \Omega_{2}^{r}$,

$$
\left\langle 1_{B}, \overline{\mathcal{Z}}_{p}^{r}(t)\right\rangle \leq \epsilon+\left\langle 1_{B^{\epsilon}}, \overline{\mathcal{Z}}^{r}(t)\right\rangle .
$$

Since $t \in[0, T]$ and $B \subset \mathbb{R}_{+}$were arbitrary, (39) and (40) imply that, almost surely on $\Omega_{1}^{r} \cap \Omega_{2}^{r}$,

$$
\sup _{t \in[0, T]} \mathbf{d}\left[\overline{\mathcal{Z}}^{r}(t), \overline{\mathcal{Z}}_{p}^{r}(t)\right] \leq \epsilon
$$

Proof of Theorem 11. By (33),$\overline{\mathcal{Z}}_{p}^{r}(\cdot) \Rightarrow \mathcal{Z}^{*}(\cdot)$ as $r \rightarrow \infty$. Theorem 1 thus follows from Theorem 2 and the converging together lemma (see e.g. Theorem 4.1 in [3]).

\section{Acknowledgement}

We are grateful to Rami Atar for suggesting this project and conjecturing the result. 


\section{References}

[1] Addie, R., And McNickle, D. Comparing different approaches to the use of DiffServ in the internet. In IEEE TENCON 2005 (2005), IEEE, pp. 1-6.

[2] Bansal, N., and Harchol-Balter, M. Analysis of SRPT scheduling: investigating unfairness. In Proceedings of ACM Sigmetrics 2001 (New York, 2001), ACM Press, pp. 279-290.

[3] Billingsley, P. Convergence of Probability Measures. John Wiley \& Sons, Inc. New York, 1968.

[4] Down, D. G., Gromoll, H. C., And Puha, A. L. Fluid limits for shortest remaining processing time queues. Mathematics of Operations Research 34 (2009), 880-911.

[5] Down, D. G., Gromoll, H. C., and Puha, A. L. State-dependent response times via fluid limits in shortest remaining processing time queues. SIGMETRICS Perform. Eval. Rev. 37, 2 (2009), 75-76.

[6] Gromoll, H. C., Kruk, Ł., And Puha, A. L. Diffusion limits for shortest remaining processing time queues. Preprint (2010). arXiv:1005.1035v1 [math.PR].

[7] Harchol-Balter, M., Sigman, K., and Wierman, A. Asymptotic convergence of scheduling policies with respect to slowdown. Performance Evaluation 49 (2002), 241-256.

[8] Nuyens, M., And ZwART, B. A large-deviations analysis of the GI/GI/1 SRPT queue. Queueing Systems 54, 2 (2006), 85-97.

[9] Prohorov, Y. V. Convergence of random processes and limit theorems in probability theory. Theory of Probability and its Applications 1 (1956), 157-214.

[10] Schrage, L. A proof of the optimality of the shortest remaining processing time discipline. Operations Research 16, 3 (1968), 687-690.

[11] SchreIBER, F. Properties and applications of the optimal queueing strategy SRPT - a survey. Archiv für Elektronik und Übertragungstechnik 47 (1993), 372-378. 
[12] Smith, W. Various optimizers for single-stage production. Naval Res. Logistics Quart. 3 (1956), 59-66.

[13] Wierman, A., And Nuyens, M. Scheduling despite inexact jobsize information. In SIGMETRICS '08: Proceedings of the 2008 ACM SIGMETRICS international conference on Measurement and modeling of computer systems (New York, NY, USA, 2008), ACM, pp. 25-36.

Department of Mathematics

UNIVERSITY OF VIRGINIA

Charlottesville, VA 22904

E-MAIL: gromoll@virginia.edu mpk2v@virginia.edu 